\title{
EKF modeling of field oriented control of six-phase induction motor
}

\author{
Asghar Taheri ${ }^{\mathrm{a}}$ \\ Unversity of Zanjan, Zanjan, Iran \\ a)taheri@znu.ac.ir
}

Abstract: This paper deals with using Extended Kalman Filter $(\mathrm{EKF})$ as a powerful tool in estimate of parameters and states of sixphase induction motor (6PIM). The speed-sensorless field-oriented control of 6PIM supplying by SVPWM is used to estimate of parameters in parameter variations. The EKF algorithm estimate stator flux, stator current, and stator resistance by measurement of stator currents of inverter. The estimation algorithms based on the stator fluxes is tested under load variations in the high velocity. The simulation results presented in this paper explain the performance of proposed method in the six-phase induction machine drive.

Keywords: EKF, six-phase induction motor, FOC

Classification: Electronic instrumentation and control

\section{References}

[1] M. A. Fnaiech, F. Betin, G. A. Capolino, and F. Fnaiech, "Fuzzy Logic and Sliding-Mode Controls Applied to Six-Phase Induction Machine With Open Phase," IEEE Trans. Ind. Electron., vol. 57, no. 1, pp. 354-364, Jan. 2010.

[2] R. Bojoi, F. Farina, A. Tenconi, F. Profumi, and E. Levi, "Dual threephase induction motor drive with digital current control in the stationary reference frame," IET J. Power Eng., vol. 20, no. 3, pp. 40-43, June/July 2006.

[3] R. Bojoi, M. Lazzari, F. Profumo, and A. Tenconi, "Digital field-oriented control for dual three-phase induction motor drives," IEEE Trans. Ind. Appl., vol. 39, no. 3, pp. 1243-1254, May/June 2003.

[4] M. Barut, S. Bogosyan, and M. Gokasan, "Speed-sensorless estimation for induction motors using extended Kalman filters," IEEE Trans. Ind. Electron., vol. 54, no. 1, pp. 272-280, Feb. 2007.

[5] E. S. de Santana, E. Bim, and W. C. do Amaral, "A Predictive Algorithm for Controlling Speed and Rotor Flux of Induction Motor," IEEE Trans. Ind. Electron., vol. 55, no. 12, pp. 4398-4407, Dec. 2008.

[6] J. Holtz, "Sensorless control of induction machines with or without signal injection," IEEE Trans. Ind. Electron., vol. 53, no. 1, pp. 7-30, Feb. 2006.

[7] Y. Zhao and T. A. Lipo, "Space Vector PWM Control of Dual Three-phase Induction Machine Using Vector Space Decomposition," IEEE Trans. Ind. Appl., vol. 31, no. 5, pp. 1100-1109, 1995.

[8] K. Marouani, L. Baghli, D. Hadiouche, A. Kheloui, and A. Rezzoug, "A New PWM Strategy Based on a 24-Sector Vector Space Decomposition 


\section{Introduction}

Recently, some advantages of multi-phase machines of various types are caused their possible attention in some applications. These machines have some advantages to three or one phase machines like higher redundancy in fault conditions that these machines can be worked in inter-phase short circuits and open circuits of some phases (one phase to three phases) [1,2]. Multiphase induction machines are used in the field of high marine propulsion, aerospace applications, and railway traction $[2,3]$. The six-phase induction machine is very interesting and is one of the most discussed types of multiphase machines. The extended kalman filter is powerful in estimation of past, present, and future states of nonlinear systems. It can be used to estimate of varied parameters and outputs while there is not any sensor to measure. To estimate of parameters, some of the systems outputs are measured and calculated by sensors, then estimation error are deduced and kalman filter coefficients are improved. If sensors have not enough accuracy to measure of some parameters, EKF will increase the accuracy of measurements. Kalman Filter and EKF are used in many papers to estimate of motor parameters and outputs of servo systems $[4,5]$. EKF is a suitable instrument to estimate of machine parameters because of stochastic properties of induction motor. Also it can be used to control of machine when machine parameters are changed. This paper presents a speed sensorless FOC of 6PIM in high speed. Estimation of velocity is powerful instrument in velocity control.

\section{Six-phase induction motor model}

The popular method in six-phase induction machine modeling is VSD (Vector Space Decomposition). In VSD method, the machine modeling is achieved in three two-dimensional orthogonal subspaces [7]. Fundamental harmonic of the machine, which produces electromechanical energy conversion and the harmonics of the order $12 n \pm 1(n=1,2,3, \ldots)$ are mapped to the $(d-q)$ subspace. Harmonics of the order $6 n \pm 1(n=1,3,5, \ldots)$ are mapped to $\left(z_{1}-z_{2}\right)$ subspace and produces losses and then they must be reduced to improve efficiency. Third harmonics are mapped to $\left(o_{1}-o_{2}\right)$ subspace. Six-phase induction motor model can be described by the following set of differential equations:

$$
\begin{aligned}
& \left\{\begin{array}{l}
V_{d q}=R_{s} I_{d q_{s}}+\rho \Psi_{d q_{s}} \pm \omega_{e} \Psi_{d q_{s}} \\
0=R_{r} I_{d q_{r}}+\rho \Psi_{d q_{r}} \pm j\left(\omega_{e}-\omega_{r}\right) \Psi_{d q_{s}}
\end{array}\right. \\
& \Psi_{d q_{s}}=L_{s} I_{d q_{s}}+M I_{d q_{r}} \\
& \Psi_{d q_{r}}=L_{r} I_{d q_{r}}+M I_{d q_{s}}
\end{aligned}
$$


Where:

$$
\begin{aligned}
& \omega_{r}=\frac{\mathrm{P}}{2} \omega_{m}, \quad L_{s}=L_{l s}+M, \\
& L_{R}=L_{l R}+M, \quad M=3 L_{m s} \\
& v_{z 1, z 2}=R_{s} i_{z 1, z 2}+L_{l s} \frac{d i_{z 1, z 2}}{d t}
\end{aligned}
$$

In FOC of 6 PIM $\psi_{r q}=0$, thus by (1) and (2) we can say:

$$
\begin{gathered}
I_{r q}=-\frac{M}{L_{R}} I_{s q}, \quad \psi_{r d}=\frac{M I_{s d}}{1+S T_{R}} \\
\omega_{s}=-\frac{R_{r} I_{r q}}{\psi_{r d}}=\frac{M}{T_{R}} \frac{I_{s q}}{\psi_{r d}} \\
\omega_{e}=\omega_{s}+\omega_{r m} \quad \theta_{e}=\int \omega_{e} d t
\end{gathered}
$$

Electromechanical energy conversion is as the follow:

$$
T_{e}=\frac{3 P}{2} I_{m}\left(\overline{\Psi_{r}} \cdot{\overline{I_{r}}}^{*}\right)
$$

Dynamic model of 6PIM in the stationary reference frame is as follow:

$$
\left\{\begin{array}{l}
X^{\circ}=A(x) X+B U+w_{1} \\
Y=C X+w_{2}
\end{array}\right.
$$

Where $X$ is the state of the system that $X=\left[\begin{array}{llll}I_{\alpha_{s}} & I_{\beta_{s}} & \psi_{\alpha_{s}} & \psi_{\beta_{s}}\end{array}\right], Y$ is the measurement parameters vector that $Y=\left[\begin{array}{ll}I_{\alpha_{s}} & I_{\beta_{s}}\end{array}\right]$, input vector is $U, U=$ $\left[u_{\alpha_{s}} u_{\beta_{s}}\right], w_{1}$ is process noise, and $w_{2}$ is measurement noise.

EKF is a suitable instrument to estimate of state-space variables and outputs. With estimate of variables, the accuracy of control system is increased. Also parameter estimation reason to reduce of measurement sensors, thus the hardware and cost is reduced. Parameters estimation is possible by measurement of uncertainty inherent of machine [5] and immeasurable parameters. EKF requires to the linearized approximation of the nonlinear model and uses the estimation of states and inputs in the linearization model. EKF model of six-phase induction motor can be indicated by recursive equations as below:

$$
\begin{gathered}
P=A_{d}(\hat{x}) P_{h} A_{d}(x)+B_{d} w_{u} B_{d}(u)+Q \\
\left.P_{h}=P-P^{*} C^{T}\left(C^{*} P\right)^{*} C^{T}+R\right)^{-1} C^{*} P \\
\left.\widehat{x}=A(\widehat{x}) * \widehat{x}+B u^{T}+P_{h} C^{T} R^{-1}\left(Y_{y}^{\prime}-C\right) * \widehat{x}\right)
\end{gathered}
$$

That

$$
A_{d}(\widehat{x})=\frac{\partial A(\widehat{x})}{\partial \widehat{x}}, \quad B_{d}(u)=\frac{\partial B(u)}{\partial u}
$$

$Q$ is the covariance matrix of the system noise, $R$ is the covariance matrix of the measurement noise, and $\mathrm{w}_{\mathrm{u}}$ is the covariance matrix of the input noise. 
EKF has two separated steps, the first step is prediction and the second one is parameters estimation. In prediction step $A(\widehat{x}), A_{d}(\widehat{x})$, and $P$ are calculated and then in estimation step $P_{h}, \hat{x}_{h}$ are calculated.

EKF estimate 6PIM state variables by using measurement of motor phase currents. DC voltage of inverter input is measured by voltage sensor, and phase voltages are computed by switching times easily. EKF based on Taylor series approximation is a stochastic state estimator that can be used to estimate of 6PIM non-linear states by a recursive algorithm. Parameter estimation of speed and machine resistance in 6PIM cause increasing of accuracy and reducing sensor in velocity measurement. 6PIM has nonlinear and uncertainty parameters as IM that are suitable to the stochastic make up of EKF [6]. EKF has some special properties as: high accuracy and low convergence time in parameter estimation and online estimate of states.

\section{Results}

In order to validate the efficiency of the proposed method, a computer simulation model as Fig. 1 is implemented using Matlab. 6PIM with fallowing parameters is used: Number of pole pairs $p=4$, stator resistance is $15 \Omega$, rotor resistance is $7.9 \Omega, \Psi_{s N}=0.55 w e b, \mathrm{~N}=1400 \mathrm{rpm}$. The controller parameters $(K p, K i)$ are chosen to achieve the best transient performance of step change in speed.

Simulation results of EKF modeling of FOC 6PIM are shown in Figs. 2 to 4 . In the simulations, motor speed reference is $100 \mathrm{rad} / \mathrm{sec}$. load torque is $0.5 \mathrm{~N}$.m in beginning and changed to $1.5 \mathrm{~N} . \mathrm{m}$ at $0.8 \mathrm{th}$. Results of this simulation are in Fig. 2. If $R_{s}$ is changed as shown in Fig. 3-a, it causes ignore in estimation algorithm, we have an error in estimation of parameters. Red line is simulation results of torque estimation and blue line is actual value of torque. Estimation of $R_{s}$ is deduced to increase accuracy in estimation. As see from Figs. 2 and 4, estimation of load torque and stator flux has good response in parameters changes. Variation of stator resistance in various states is shown in Fig. 3-a. as see from this figure resistance is changed from $15 \Omega$ to $19.5 \Omega$ in 0.6 th second and remained in that value. As see from Fig. 4 b estimation of $R_{s}$ has a big oscillation in beginning. Suitable selection of initial value can decrease this oscillation. If covariance matrix is changed to

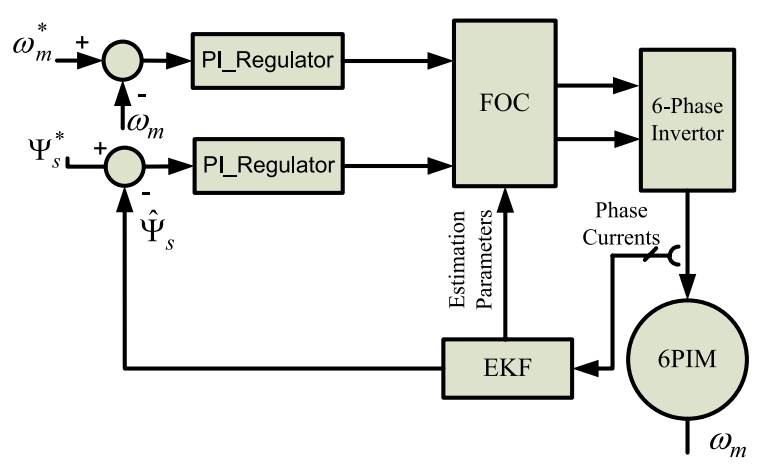

Fig. 1. Block diagram of simulation model. 


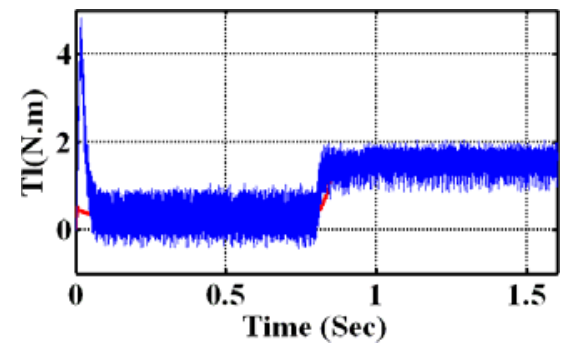

(a)

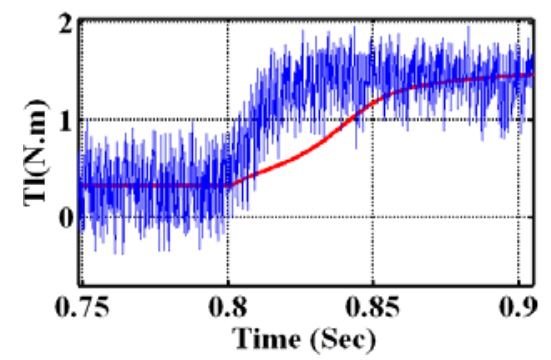

(c)

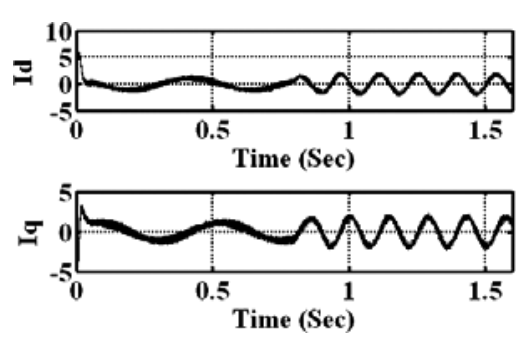

(b)

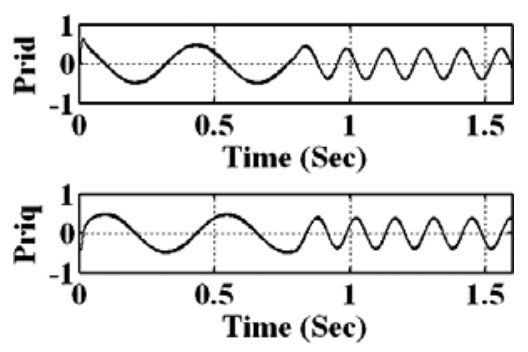

(d)

Fig. 2. EKF modeling of FOC six-phase induction motor, (a) Load torque and estimation of load, (b) stator current in $d-q$ axes, (c) enlarged of load torque, (d) rotor flux in $d-q$ axes.

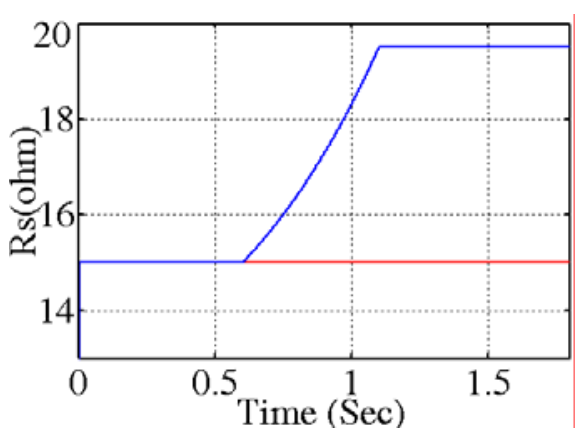

(a)

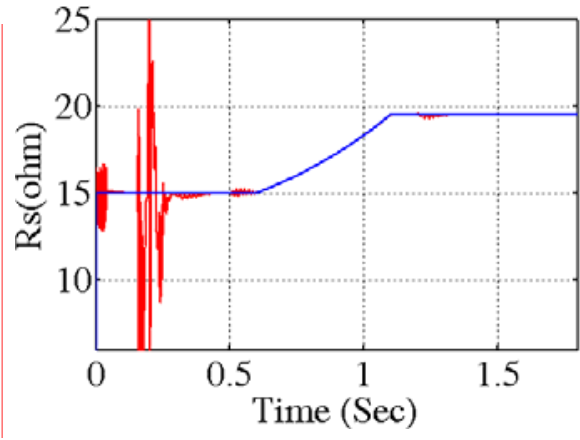

(b)

Fig. 3. (a) Change of $R_{s}$ in estimation algorithm, (b) estimation of $R_{s}$ in high speed.

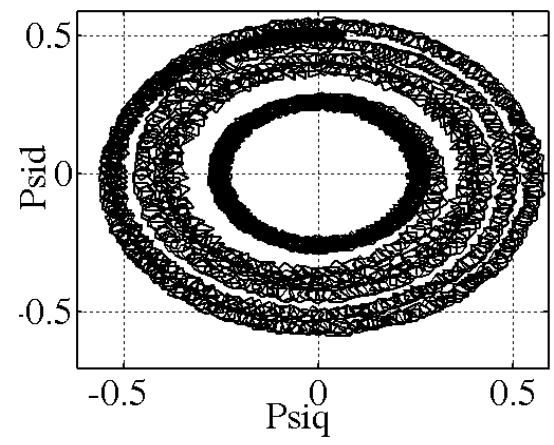

(a)

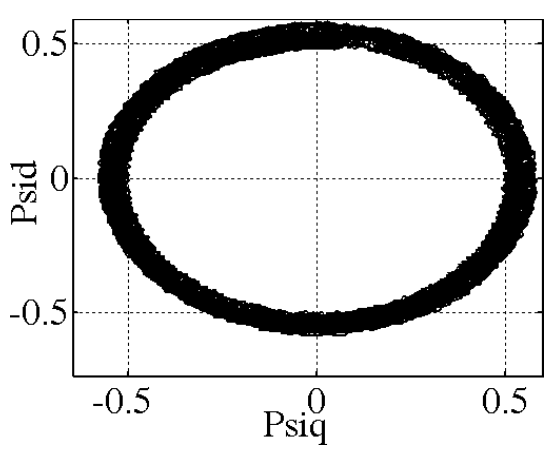

(b)

Fig. 4. Stator flux in $d$ axis vs. $q$ axis when motor speed is high, (a) without estimation of $R_{s}$, (b) with estimation of $R_{s}$. 
an optimal value, this oscillation can be reduced. In Fig. 4 stator flux in $d$ axis vs. $q$ axis with and without estimation of stator resistance are shown. As shown from Fig. 4-b, stator flux with estimation of stator resistance has a good-looking shape with low noise.

\section{Conclusion}

In this paper EKF sensorless estimation algorithm is simulated for FOC of 6PIM. The motor is derived by SVPWM to have minimum loss in $\left(z_{1}-z_{2}\right)$ domain. EKF estimation of stator currents, stator resistance, and stator flux have good responses in parameters changes. Ignoring stator resistance variations causes large error in parameters estimation and instability in control system. 